\title{
Convalescent Plasma Therapy for COVID-19: Current Status and Future Directions
}

\author{
Jayanth Seshan ${ }^{1}$ Surya K. Dube ${ }^{1, \odot}$ Vanitha Rajagopalan ${ }^{1, \odot}$ Pragyan S. Panda ${ }^{2}$ Girija P. Rath
}

\author{
${ }^{1}$ Department of Neuroanaesthesiology and Critical Care, \\ Neurosciences Center, All India Institute of Medical Sciences, \\ New Delhi, India \\ ${ }^{2}$ Department of Microbiology, Janakpuri Super Speciality Hospital \\ Society, Janakpuri, New Delhi, India
}

\begin{abstract}
Address for correspondence Surya K. Dube, MD, DM, Department of Neuroanaesthesiology and Critical Care, Neurosciences Center, All India Institute of Medical Sciences, Ansari Nagar, New Delhi 110029, India (e-mail: surya.dube@yahoo.co.in).
\end{abstract}

J Neuroanaesthesiol Crit Care:2020;7:140-147

\begin{abstract}
Keywords

- convalescent plasma

- coronavirus

- COVID-19

- immunotherapy

- SARS-CoV-2

The ongoing pandemic of coronavirus disease 2019 (COVID-19) has triggered a global health crisis probably due to a lack of a reliable cure till date. Several clinical trials are ongoing, but initial results have not been overly promising. Convalescent plasma (CP), which refers to plasma collected from individuals recovered from an illness and developed antibodies against the pathogen, is also being proposed as a therapeutic option for COVID-19 treatment in severe cases to achieve short-term immunity against the virus. Use of $\mathrm{CP}$ is not new, and it has been used in various outbreaks over the past century, ranging from the Spanish influenza outbreak in 1918 to the recent Middle East respiratory syndrome (MERS). However, data available on its use in COVID-19 patients is limited. Use of CP so far is restricted to a "rescue therapy" and needs further trials to assess its possible use in other situations (prevention, postexposure prophylaxis) and patient populations (considering age and comorbid illnesses). In this review, we will try to summarize the current status of use of CP for COVID-19 and ongoing trials in India and elsewhere and will discuss the possible avenues for its use in future.
\end{abstract}

\section{Introduction}

Novel coronavirus disease 2019 (COVID-19) has become a pandemic now. The spectrum of clinical presentation of COVID-19 ranges from usual respiratory symptoms to more serious life-threatening events such as acute respiratory distress syndrome (ARDS), shock, cardiac injury, thromboembolic phenomenon, and even death. ${ }^{1,2}$ The uncertain pathophysiology and varied clinical course are the major hurdles in its management. Various pharmacological options (antiviral, anti-inflammatory agents, alone or in combination, monoclonal antibodies) have been tried so far with variable results without any definitive evidence on the efficacy of any of these agents. Other supportive treatments currently employed are oxygen supplementation, vasopressors, and mechanical ventilation, and extracorporeal membrane oxygenation ([ECMO] for severe cases).

Dol https://doi.org/ 10.1055/s-0040-1716594 ISSN 2348-0548.
Passive immunotherapy with convalescent plasma (CP) from recovered COVID-19 cases is now being explored as a treatment option for those cases classified as critical (respiratory failure, septic shock, multiorgan dysfunction). ${ }^{3}$ Passive immunotherapy involves administration of antigen-specific antibodies in an individual to achieve short-term immunity against the said pathogen to eradicate it from the bloodstream. CP has been used in the treatment of patients during previous outbreaks of coronaviruses (Middle-East respiratory syndrome [MERS] and severe acute respiratory syndrome [SARS]), H1N1 and H5N1 influenza A pandemics, as well as in hemorrhagic fevers caused by Ebola and Junín virus. ${ }^{4-9}$ Many ongoing studies are trying to explore the role of $\mathrm{CP}$ in COVID-19 cases, but its efficacy in COVID-19 management is still unclear. This review aims to provide the readers with an overview of available evidence for the use of $\mathrm{CP}$ in severe acute respiratory illness (SARI) of viral etiology and information about the trials on CP use in COVID-19 patients. 


\section{Rationale for Convalescent Plasma}

Convalescent blood or blood products are collected from an individual recently cured of a disease and developed humoral immunity against the pathogen, hence serve as the human source for specific antibodies. The presence of high titers of neutralizing antibodies in patients who recovered from the viral infection and the absence of such antibodies to the novel virus in the general population forms the basis of this treatment. The proposed mode of action is the rapid reduction in viremia ( $<48$ hours following transfusion) followed by suppression of the proinflammatory state. This helps in rapid patient recovery from various complications such as ARDS. Use of CP in COVID-19 reduces the chances of emergence of antiviral drug resistance, and the polyclonal nature of these antibodies minimizes the risk of escape mutant (which can occur with monoclonal antibody treatment) and faster reduction of viremia as compared with antiviral medications.

\section{Selection of Donors}

During the previous SARS outbreak, the criteria adopted for $\mathrm{CP}$ donor selection included afebrile status for at least 7 days, chest radiographic improvement by at least $25 \%$, no requirement of oxygen supplementation, and a minimum of 14 days following symptom onset. ${ }^{5}$ Similar criteria may be chosen for the present pandemic of COVID-19 because both outbreaks are caused by coronavirus, which primarily affects the respiratory system, progressing eventually to involve multiple system dysfunction. The most recent evidence available for $\mathrm{CP}$ in COVID-19 is a randomized trial comparing plasma therapy with standard treatment alone in 103 patients wherein laboratory-confirmed (reverse transcriptase-polymerase chain reaction [RT-PCR]) cases of COVID-19, fully recovered and discharged from hospital for more than 2 weeks with at least two negative follow-up RT-PCR, were considered for plasma donation. ${ }^{3}$ Similar criteria have been proposed by other authors as well. ${ }^{10}$ In a recent pilot study on COVID-19 patients, the donors chosen were the patients who were 3 weeks postsymptom onset and 4 days postdischarge. ${ }^{11}$ The patient and donor eligibility criteria for CP therapy taken out from the U.S. Food and Drug Administration (FDA) guidelines are summarized in - Table 1. This FDA guideline has recommendations for health care providers and investigators on the administration and study of investigational $\mathrm{CP}$ collected from individuals who have recovered from COVID-19. It also guides on the topics of pathways of use of $\mathrm{CP}$, patient eligibility, collection of plasma, labeling, and record-keeping. ${ }^{12}$ However, these FDA guidelines provide only information on the use of plasma and do not elaborate on indications for use, dosage, contraindications, or cautions. Nevertheless, it is imperative for the donors to be seropositive for coronavirus and screened negative for hepatitis B and C, HIV, and syphilis. It is prudent to follow previous World Health Organization (WHO) recommendations for the selection of blood donors considering important factors such as age, anemia, obesity, and other comorbidities. ${ }^{13}$ Informed consent of the donor for $\mathrm{CP}$ donation is mandatory.

\section{Plasma Collection}

Collection of plasma by apheresis technique and its use as a therapeutic pool is preferred as it allows for larger volumes of collection, more frequent donations ( 2 weekly intervals), and the possibility of reinfusion of red blood cells to donors, which eliminates any risk of lowering donor hemoglobin levels. ${ }^{14}$ The allowable plasma volume collection by apheresis is $625 \mathrm{~mL}$ for donors, with a bodyweight of 50 to $80 \mathrm{~kg}$, and it can be performed at 2 weekly intervals. ${ }^{15}$ Although a recent FDA statement has termed guidelines for volume collection obsolete, it is preferable to restrict to this limit in the current scenario. ${ }^{16}$ This short time interval between two donations ( 2 weeks), when compared with the interval of 3 months for whole blood donations, is advantageous, although large-scale availability of apheresis machines and trained operators pose major obstacles. ${ }^{17}$ Similar to fresh frozen plasma, COVID-19 CP should be frozen within 8 hours after collection, stored in aliquots of $200 \mathrm{~mL}$ each, and stored at a temperature of $-18^{\circ} \mathrm{C}$ or lower.

\section{Estimation of Antibody Titers}

Transfusion of CP containing high neutralizing antibody titer (NAT) helps to achieve an earlier and effective

Table 1 Summary of the patient and donor criteria for CP therapy ${ }^{12}$

\begin{tabular}{|l|}
\hline Patient eligibility criteria \\
- Laboratory-confirmed COVID-19. \\
- Severe or immediately life-threatening disease. Severe \\
tachypnea ( $\geq 30 /$ min), $\mathrm{SpO}_{2} \leq 93 \%, \mathrm{PaO}_{2} / \mathrm{FiO}_{2}<300$, and \\
lung infiltrates $>50 \%$ within 24 to 48 h. The presence of \\
respiratory failure, septic shock, and multiorgan dysfunction \\
or failure is defined as life-threatening. \\
- Informed consent provided by the patient or healthcare \\
proxy. \\
Donor eligibility criteria \\
- Evidence of COVID-19 documented by a laboratory test \\
either diagnostic (nasopharyngeal swab) at the time of \\
illness or positive serology (SARS-CoV-2 antibodies) after \\
recovery if prior diagnostic testing was not performed at the \\
time COVID-19 was suspected. \\
- Either complete resolution of symptoms at least 28 days \\
prior to donation or complete resolution of symptoms at \\
least 14 days prior to donation along with negative results \\
for COVID-19 either from one or more nasopharyngeal swab \\
specimens or by a molecular diagnostic test from blood. \\
- Male donors, or female donors who have not been pregnant, \\
or female donors who have been tested since their most \\
recent pregnancy and results interpreted as negative for HLA \\
antibodies. \\
- SARS-CoV-2 NAT testing: when testing is available, neutral- \\
izing antibody titers of at least $1: 160$ is recommended. A \\
titer of $1: 80$ may be considered acceptable if an alternative \\
matched unit is not available. A sample maybe stored for \\
estimation at a later date if measures for antibody titer \\
estimation are not available at the time of collection/ \\
transfusion.
\end{tabular}

Abbreviations: COVID-19, coronavirus disease 2019; CP, convalescent plasma; HLA, human leukocyte antigen; NAT, neutralizing antibody titer; SARS-CoV-2, severe acute respiratory syndrome coronavirus 2 . 
seroconversion. The titer of antibody is important as apparent from the fact that during the MERS outbreak, the use of CP did not help in clinical recovery, and one of the possible reasons suggested was the lack of proper estimation of NAT. It was hypothesized that the plasma transfused to the patients could have had low antibody titers since it was collected from cases who recovered from a mild illness and hence did not develop high antibody levels. ${ }^{4,18,19}$ The other problem with low serum antibody titers is an antibody-dependent enhancement (ADE) of the virus, which will be discussed later. ${ }^{20} \mathrm{Li}$ et al in their study on COVID-19 patients estimated the antibody titer against a spike protein on the receptor-binding domain (S-RBD) and showed that titers of at least 1:640 were required for transfusion. ${ }^{3}$ Evidence from the use of $\mathrm{CP}$ during previous viral outbreaks indicates toward the clinical efficacy of $\mathrm{CP}$ with an antibody titer of 1:160 in SARS and H1N1 influenza $A^{5,6}$ In their recommendations for investigational plasma treatment for COVID-19, the FDA suggests a NAT of $1: 160$, if the estimation is available. ${ }^{12}$

\section{Volume of Convalescent Plasma Transfusion}

Cheng et al in their study on CP in SARS patients used a mean plasma transfusion volume of $279 \mathrm{~mL}$ (range: $160-640 \mathrm{~mL} ; 4-5 \mathrm{~mL} / \mathrm{kg}$ ) with a NAT of $1: 160$ in 80 adult patients (median age: 45 years). They found that the outcome was more dependent on the time of administration than the volume infused. ${ }^{5}$ Recent studies on COVID-19 patients in China reported the transfusion of one dose of $200 \mathrm{~mL}$ of inactivated CP with neutralization activity of $>1: 640$ within 4 hours of collection. ${ }^{3,11}$ Shen et al reported the effects of plasma transfusion in five critically ill patients suffering from COVID-19, where 400 $\mathrm{mL}$ of plasma was transfused. ${ }^{21}$ A study on CP for Ebola patients used a strategy of total transfusion of 400 to 500 $\mathrm{mL}$ in two aliquots in an hour. ${ }^{22,23}$ Based on the current evidence, the use of standard aliquots of $200 \mathrm{~mL}$ of CP $(4-5 \mathrm{~mL} / \mathrm{kg})$ in adults seems to be a rational choice.

\section{Repeat Dose: When and How Much to Give?}

Ko et al reported their experience of CP transfusion in the management of the MERS outbreak in 2015. Out of the 13 patients with respiratory failure, 3 patients received $\mathrm{CP}$ and 1 patient received a repeat transfusion 1 week after the initial dose as no seroconversion was observed after the initial dose. ${ }^{4}$ Arabi et al in their study planned to transfuse 2 units of plasma (250-350 mL/unit) in MERS CoV patients after assessing the feasibility of procuring CP from donors, but their study did not progress to this phase as they did not find donors with adequate antibody titers. ${ }^{18}$ One case report during the SARS outbreak mentions the use of $500 \mathrm{~mL}$ of $\mathrm{CP},(250 \mathrm{~mL}$ transfused 12 hours apart). Despite clinical recovery with the initial dose, the second dose was also transfused as they planned to deliver $5 \mathrm{~g}$ of immunoglobulin ( $10 \mathrm{~g} / \mathrm{L}$ of plasma $){ }^{24}$

\section{Ideal Time of Administration of Convalescent Plasma}

Most viral illnesses have maximum viremia at around the end of the first week of infection followed by activation of host immune responses and viral clearance after 2 weeks. In COVID-19, after an incubation period of 2 to 12 days (mean: 5-6 days), patients develop clinical symptoms that progress to severe illness around day 8 (5-10 days of symptom onset). ${ }^{25}$ It is therefore ideal to administer CP early in the course of illness. In the study by Li et al, the median duration between symptom onset and randomization to the $\mathrm{CP}$ group was 27 days (interquartile range: 22-39). This finding is in stark contrast to all the available literature that favors the early administration of $\mathrm{CP}$ (less than 14 days).5,26 It is suggested that the lack of a significant outcome difference in this study (time to clinical improvement, discharge or mortality) compared with standard treatment is due to the delayed administration of plasmatherapy.,27

\section{Monitoring for Response to Therapy}

Assessment of response to $\mathrm{CP}$ treatment is based on various parameters like a clinical improvement (normalization of body temperature and oxygen saturation, and relief of dyspnea), changes in radiological parameters (resolution of lung lesions on chest (T), and estimation of viral load. A favorable response is usually evident within 2 to 3 days of plasma transfusion. Mair-Jenkins et al in their meta-analysis on the effectiveness of CP on SARI mentioned only one retrospective study that reported nonsignificant reductions in the duration of mechanical ventilation and ECMOfollowing $\mathrm{CP}^{28,29}$ The other published studies, they reported, did not show adequate data on critical care support and plasma therapy. Lymphocytopenia is a common finding in COVID-19 and is supposedly related to the consumption and inhibition of the host's immune function by the virus. An increase in lymphocyte count was observed after $\mathrm{CP}$ and was associated with clinical recovery. ${ }^{11,30}$ Although a reduction in viral load is evident as early as 24 hours of transfusion, its estimation is overshadowed by the more favored clinical, laboratory, and radiological recovery.

\section{Adverse Events Following CP Transfusion}

Febrile reactions and mild allergy in the form of urticaria can occur in $1 \%$ of those receiving plasma transfusions and are managed with supportive care, antihistamines, and small doses of corticosteroids if required. Risk of severe allergy and anaphylaxis is seen in $<1$ in 1,00,000 of cases. Rare complications include citrate toxicity (citrate induced hypocalcemia), transfusion-related acute lung injury (TRALI), and transfusion-associated circulatory overload. ${ }^{31}$ Although rare, significant attention needs to be paid toward TRALI as it may exacerbate the pulmonary injury caused by coronavirus. ${ }^{4}$ The other common adverse events are transient elevation of body temperature, jaundice, and phlebitis. Nonmatched ABO blood administration can lead to serious complications including anaphylaxis. ${ }^{32}$ The risk of transfusion-related transmission of 
blood pathogen is very minimal with the current screening techniques employed. A rare complication that can occur is an $\mathrm{ADE}$ of the virus, which was initially observed in the dengue vaccine recipient. It was observed that the risk of severe secondary dengue in the form of dengue hemorrhagic fever (DHF) or dengue shock syndrome (DSS) was high in those with a low antibody titer from either previous illness or vaccination. Though not demonstrated in previous viral outbreaks such as SARS, MERS, and Ebola, a theoretical risk remains with a lower antibody titer in the donor plasma, which may enhance viral penetration in the host cells and hence their replication. ${ }^{20}$

\section{Limitations of Convalescent Plasma}

None of the published studies on viral outbreaks, except one recent trial on COVID-19, has compared CP with other forms of treatment. Most of all studies mention $\mathrm{CP}$ as a last resort and given along with standard care including antiviral agents and corticosteroids in some cases, and hence the therapeutic efficacy of CP alone could not be asserted. Even the recent randomized trial was limited by its sample size due to early termination as the disease spread was contained. Limitations of treatment exist at various levels, ranging from donor selection, collection of plasma, estimation of antibody titers, transfusion into the recipient, to its effects on the patient. The selected donor must be free from the virus at the time of selection or else it exposes the health care worker to the disease during screening and collection. Convalescent plasma collection may result in a reduction in the collection of whole blood and blood products, resulting in a shortage/crisis. Given the reports of reinfection following clinical recovery and negative molecular reports, it becomes mandatory to include retesting for the virus in a potential donor even if he/she has tested negative already.

In an unexpected outbreak with a large requirement of plasma, it may not be feasible to estimate NAT as it requires biosafety level 3 laboratory and may have to rely on ELISA (enzyme-linked immunosorbent assay) for estimation immunoglobulin (Ig) $\mathrm{M} / \mathrm{IgG} .^{33}$ Accordingly, if plasma with inadequate NAT is transfused, seroconversion in recipient may not occur or produce an immediate response leading to worsening. ${ }^{4}$ There is no certainty regarding the optimal dose and dosage of plasma transfusion in COVID-19. Furthermore, there are no data or trials to support transfusions in pediatric patients.

\section{Available Literature and Current Trials}

- Table 2 provides a summary of studies on CP therapy in SARI of viral etiology. A Cochrane review on CP in COVID-19 that was recently published assessed eight studies (seven case series and one prospective single-arm intervention study) with a total of 32 participants. They identified "very low-certainty" evidence on the clinical effectiveness (weaning off respiratory support and mortality outcomes) and safety of $\mathrm{CP}$, with all included studies having low reporting quality and high risk of bias; the results of 47 ongoing studies (22 randomized controlled trials) are awaited. ${ }^{34}$ In the Indian context, a "Phase-II, Openlabel, Randomised Controlled Trial to Assess the Safety and Efficacy of Convalescent Plasma to Limit COVID-19 Associated Complications in Moderate Disease" (PLACID Trial) is currently undergoing under the Indian Council for Medical Research. This study is planned as a multicenter clinical trial with an estimated sample size of 452 and 46 participating institutions in India. This study involves administration of 2 doses of $200 \mathrm{~mL}$ each of CP to laboratory-confirmed cases of COVID-19 with moderate disease (as defined by respiratory rate $>24 /$ minute; $\mathrm{PaO}_{2}: \mathrm{FiO}_{2}$ of 200-300) and assesses the avoidance of progression to severe ARDS or mortality at 28 days. Seven other trials are ongoing at various centers in the country and are in the recruitment phase, with results awaited. Most of them are designed as randomized trials to compare the efficacy of CP transfusion with the usual standard of care..$^{35,36}$ "National COVID-19 Convalescent Plasma Project" is currently undergoing in the United States to assess the efficacy of $\mathrm{CP} .{ }^{37}$

\section{Future Directions}

The current literature on $\mathrm{CP}$ focuses mainly on its use in critically ill patients. Considering the frequent and unexpected outbreaks of viral illness in this century thus far, it is important to consider plasma as an option not only for treatment in severe cases but also for prophylaxis. Postexposure prophylaxis in the vulnerable population (health care workers, immunocompromised, individuals with comorbidities, etc.) would certainly offer clinical as well as social benefits. Trials are required to test the efficacy of plasma in patients presenting with mild/moderate illness to prevent their worsening to a stage requiring mechanical ventilation. Also, there is no evidence for its use in the pediatric population as they also form a part of the vulnerable population.

\section{Conclusion}

At present, $\mathrm{CP}$ offers scope as a rescue therapy in the current pandemic COVID-19, even though it is inadequately supported with large randomized trials. Hopefully, more evidence will be available in the near future to support or refute the safety and efficacy of $\mathrm{CP}$ not just as a rescue therapy but also as a prophylactic measure in COVID-19 management. 


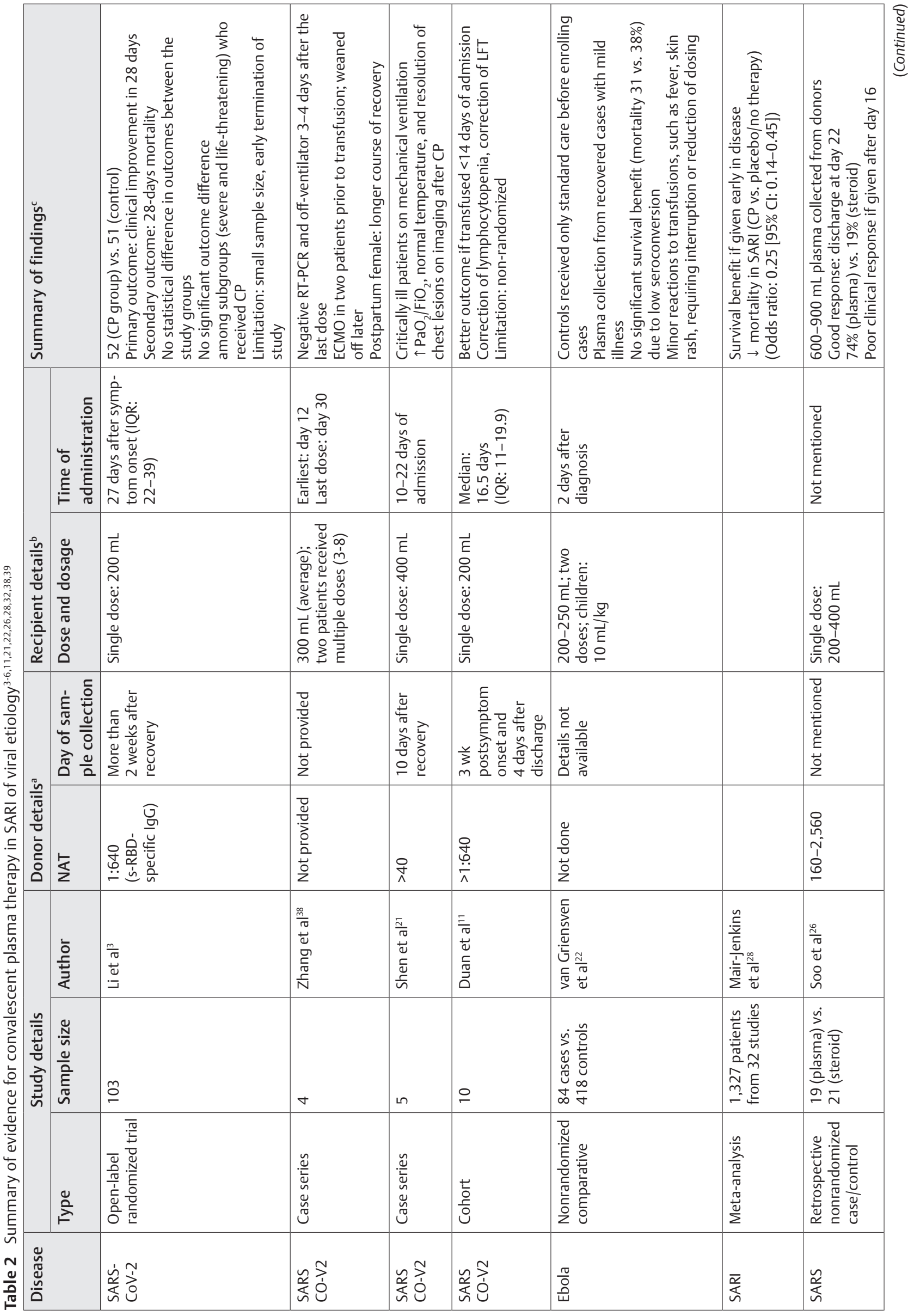


Plasma Therapy for COVID-19 Seshan et al. 145

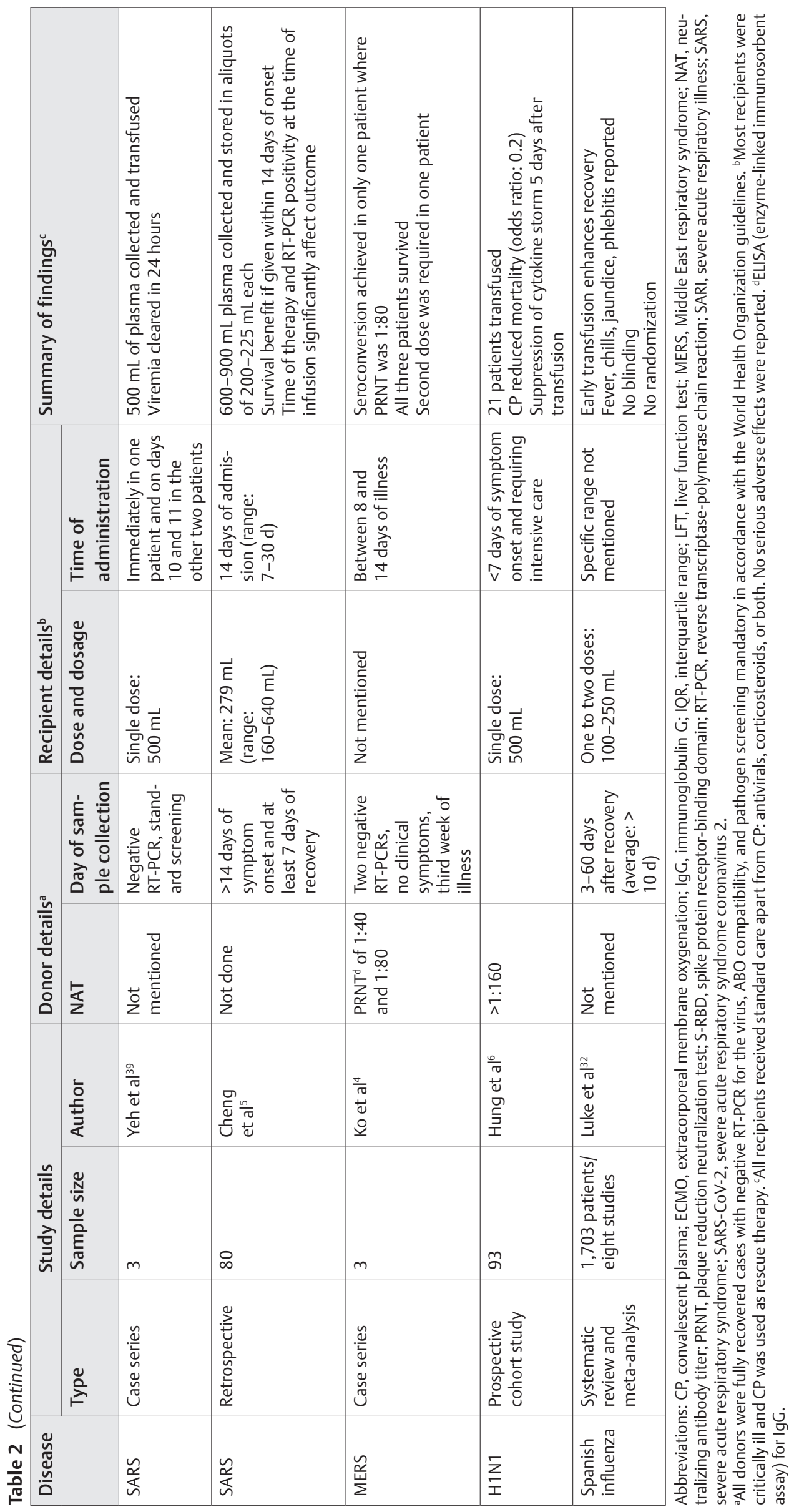




\section{Conflict of Interest}

None declared.

\section{References}

1 Huang C, Wang Y, Li X, et al. Clinical features of patients infected with 2019 novel coronavirus in Wuhan, China. Lancet 2020;395(10223) :497-506

2 Vanamoorthy P, Singh GP, Bidkar PU, et al. The Neurocritical Care Society of India (NCSI) and the Indian Society of Neuroanaesthesiology and Critical Care (ISNACC) Joint Position Statement and Advisory on the Practice of Neurocritical Care during the COVID-19 Pandemic. J Neuroanaesth Crit Care 2020;2:49-114

3 Li L, Zhang W, Hu Y, et al. Effect of convalescent plasma therapy on time to clinical improvement in patients with severe and life-threatening COVID-19: a randomized clinical trial. JAMA 2020;324(5):1-11

$4 \mathrm{Ko} \mathrm{JH}$, Seok H, Cho SY, et al. Challenges of convalescent plasma infusion therapy in Middle East respiratory coronavirus infection: a single centre experience. Antivir Ther 2018;23(7):617-622

5 Cheng Y, Wong R, Soo YO, et al. Use of convalescent plasma therapy in SARS patients in Hong Kong. Eur J Clin Microbiol Infect Dis 2005;24(1):44-46

6 Hung IF, To KK, Lee CK, et al. Convalescent plasma treatment reduced mortality in patients with severe pandemic influenza $\mathrm{A}$ (H1N1) 2009 virus infection. Clin Infect Dis 2011;52(4):447-456

7 Zhou B, Zhong N, Guan Y. Treatment with convalescent plasma for influenza A (H5N1) infection. N Engl J Med 2007;357(14):1450-1451

8 Sahr F, Ansumana R, Massaquoi TA, et al. Evaluation of convalescent whole blood for treating Ebola Virus Disease in Freetown, Sierra Leone. J Infect 2017;74(3):302-309

9 Ruggiero HA, Pérez Isquierdo F, Milani HA, et al. Treatment of Argentine hemorrhagic fever with convalescent's plasma. 4433 cases [in French]. Presse Med 1986;15(45): 2239-2242

10 Bloch EM, Shoham S, Casadevall A, et al. Deployment of convalescent plasma for the prevention and treatment of COVID-19. J Clin Invest 2020;130(6):2757-2765

11 Duan K, Liu B, Li C, et al. Effectiveness of convalescent plasma therapy in severe COVID-19 patients. Proc Natl Acad Sci U S A 2020;117(17):9490-9496

12 U.S. Food and Drug Administration. Recommendations for Investigational COVID-19 Convalescent Plasma. Available at: https://www.fda.gov/vaccines-blood-biologics/investigational-new-drug-ind-or-device-exemption-ide-process-cber/recommendations-investigational-covid-19-convalescent-plasma. Accessed April 29, 2020

13 World Health Organization. Blood donor selection: guidelines on assessing donor suitability for blood donation. Available at: https://apps.who.int/iris/handle/10665/76724. Accessed June 5, 2020

14 Marano G, Vaglio S, Pupella S, et al. Convalescent plasma: new evidence for an old therapeutic tool? Blood Transfus 2016;14(2):152-157

15 Williams AE. FDA Considerations Regarding Frequent Plasma Collection Procedures. Available at: https://www. ihn-org.com/wp-content/uploads/2019/08/4-Williams FreqPlasma-2-21-131.pdf. Accessed June 5, 2020

16 U.S. Food and Drug Administration. Compliance Policy Guide (CPG): CPG Sec. 252.110 Volume Limits for Automated Collection of Source Plasma (Obsolete, Withdrawn on 11/28/2017).
Available at: https://www.fda.gov/regulatory-information/ search-fda-guidance-documents/cpg-sec-252110-volum e-limits-automated-collection-source-plasma-obsolete-withdrawn-11282017. Accessed April 27, 2020

17 Wong HK, Lee CK, Hung IFN, et al. Practical limitations of convalescent plasma collection: a case scenario in pandemic preparation for influenza A (H1N1) infection. Transfus (Paris) 2010;50(9):1967-1971

18 Arabi YM, Hajeer AH, Luke T, et al. Feasibility of using convalescent plasma immunotherapy for MERS-CoV infection, Saudi Arabia. Emerg Infect Dis 2016;22(9):1554-1561

19 Jh K, Ma MHS, Ge P, et al. Serologic responses of 42 MERScoronavirus-infected patients according to the disease severity. Diagn Microbiol Infect Dis 2017;89(2):106-111

20 Katzelnick LC, Gresh L, Halloran ME, et al. Antibody-dependent enhancement of severe dengue disease in humans. Science 2017;358(6365):929-932

21 Shen C, Wang Z, Zhao F, et al. Treatment of 5 critically ill patients with COVID-19 with convalescent plasma. JAMA 2020;323(16):1582-1589

22 van Griensven J, Edwards T, de Lamballerie X, et al. Evaluation of convalescent plasma for Ebola virus disease in Guinea. N Engl J Med 2016;374(1):33-42

23 World Health Organization. Use of convalescent whole blood or plasma collected from patients recovered from Ebola virus disease. Available at: https://www.who.int/csr/resources/publications/ebola/convalescent-treatment/en/. Accessed June 4, 2020

24 Kong L. Severe acute respiratory syndrome (SARS) Transfus Apher Sci. 2003;29(1):101

25 Johns Hopkins Medicine. Coronavirus COVID-19. Available at: https://www.hopkinsguides.com/hopkins/.//view/Johns_ Hopkins_ABX_Guide/540747/all/Coronavirus_COVID_19_SARS_CoV_2?refer=true. Accessed April 27, 2020

26 Soo YO, Cheng Y, Wong R, et al. Retrospective comparison of convalescent plasma with continuing high-dose methylprednisolone treatment in SARS patients. Clin Microbiol Infect 2004;10(7):676-678

27 Casadevall A, Joyner MJ, Pirofski LA. A randomized trial of convalescent plasma for COVID-19-potentially hopeful signals. JAMA 2020. Doi: 10.1001/jama.2020.10218

28 Mair-Jenkins J, Saavedra-Campos M, Baillie JK, et al. The effectiveness of convalescent plasma and hyperimmune immunoglobulin for the treatment of severe acute respiratory infections of viral etiology: a systematic review and exploratory meta-analysis. J Infect Dis 2015;211(1): $80-90$

29 Chan KK, Lee KL, Lam PKN, Law KI, Joynt GM, Yan WW. Hong Kong's experience on the use of extracorporeal membrane oxygenation for the treatment of influenza A (H1N1) Hong Kong Med J 2010;16(6):447-454

30 Chen N, Zhou M, Dong X, et al. Epidemiological and clinical characteristics of 99 cases of 2019 novel coronavirus pneumonia in Wuhan, China: a descriptive study. Lancet 2020;395(10223):507-513

31 Hellstern P, Muntean W, Schramm W, Seifried E, Solheim BG. Practical guidelines for the clinical use of plasma. Thromb Res 2002;107:S53-S57

32 Luke TC, Kilbane EM, Jackson JL, Hoffman SL. Metaanalysis: convalescent blood products for Spanish influenza pneumonia: a future H5N1 treatment? Ann Intern Med 2006;145(8):599-609

33 Mourya DT, Yadav PD, Majumdar TD, Chauhan DS, Katoch VM. Establishment of Biosafety Level-3 (BSL-3) laboratory: important criteria to consider while designing, constructing, 
commissioning \& operating the facility in Indian setting. Indian J Med Res 2014;140(2):171-183

34 Valk SJ, Piechotta V, Chai KL, et al. Convalescent plasma or hyperimmune immunoglobulin for people with COVID-19: a rapid review. Cochrane Database Syst Rev 2020;5(5):CD013600

35 CTRI. A Phase II, Open Label, Randomized Controlled Trial to Assess the Safety and Efficacy of Convalescent Plasma to Limit COVID-19 Associated Complications in Moderate Disease. Available at: http://ctri.nic.in/Clinicaltrials/showallp.php? $\operatorname{mid} 1=43149 \&$ EncHid=\&userName=convalescent $\% 20$ plasma. Accessed May 25, 2020

36 Search Result, Clinical Trials Registry - India (CTRI) [Internet]. [cited 2020 May 25]. Available at: http://ctri.nic.in/ Clinicaltrials/pubview2.php. Accessed May 25, 2020
37 National COVID-19 Convalescent Plasma Project. Component 3: Clinical Trials. Available at: https://ccpp19.org/healthcare_ providers/component_3/index.html. Accessed May 25, 2020

38 Zhang B, Liu S, Tan T, et al. Treatment with convalescent plasma for critically Ill patients with SARS-CoV-2 infection. Chest 2020;158(1):e9-e13

39 Yeh KM, Chiueh TS, Siu LK, et al. Experience of using convalescent plasma for severe acute respiratory syndrome among healthcare workers in a Taiwan hospital. J Antimicrob Chemother 2005;56(5):919-922 